\title{
Monitoring of malaria parasite resistance to chloroquine and sulphadoxine-pyrimethamine in the Solomon Islands by DNA microarray technology
}

Marie Ballif1,2, Jeffrey Hii ${ }^{3}$, Jutta Marfurt ${ }^{1,2}$, Andreas Crameri ${ }^{1,2}$, Adam Fafale ${ }^{4}$, Ingrid Felger ${ }^{1,2}$, Hans-Peter Beck ${ }^{1,2}$, Blaise Genton ${ }^{1,2,5^{*}}$

\begin{abstract}
Background: Little information is available on resistance to anti-malarial drugs in the Solomon Islands (SI). The analysis of single nucleotide polymorphisms (SNPs) in drug resistance associated parasite genes is a potential alternative to classical time- and resource-consuming in vivo studies to monitor drug resistance. Mutations in pfmdr1 and pfcrt were shown to indicate chloroquine (CQ) resistance, mutations in pfdhfr and pfdhps indicate sulphadoxine-pyrimethamine (SP) resistance, and mutations in pfATPase6 indicate resistance to artemisinin derivatives.
\end{abstract}

Methods: The relationship between the rate of treatment failure among 25 symptomatic Plasmodium falciparuminfected patients presenting at the clinic and the pattern of resistance-associated SNPs in P. falciparum infecting 76 asymptomatic individuals from the surrounding population was investigated. The study was conducted in the SI in 2004. Patients presenting at a local clinic with microscopically confirmed $P$. falciparum malaria were recruited and treated with CQ+SP. Rates of treatment failure were estimated during a 28-day follow-up period. In parallel, a DNA microarray technology was used to analyse mutations associated with $C Q, S P$, and artemisinin derivative resistance among samples from the asymptomatic community. Mutation and haplotype frequencies were determined, as well as the multiplicity of infection.

Results: The in vivo study showed an efficacy of $88 \%$ for CQ+SP to treat $P$. falciparum infections. DNA microarray analyses indicated a low diversity in the parasite population with one major haplotype present in $98.7 \%$ of the cases. It was composed of fixed mutations at position 86 in pfmdr1, positions 72, 75, 76, 220, 326 and 356 in pfcrt, and positions 59 and 108 in pfdhfr. No mutation was observed in pfdhps or in pfATPase6. The mean multiplicity of infection was 1.39.

Conclusion: This work provides the first insight into drug resistance markers of $P$. falciparum in the SI. The obtained results indicated the presence of a very homogenous $P$. falciparum population circulating in the community. Although CQ+SP could still clear most infections, seven fixed mutations associated with CQ resistance and two fixed mutations related to SP resistance were observed. Whether the absence of mutations in pfATPase6 indicates the efficacy of artemisinin derivatives remains to be proven.

\footnotetext{
* Correspondence: blaise.genton@unibas.ch

'Swiss Tropical and Public Health Institute, Department of Medical Parasitology and Biology of Infection, Department of Epidemiology and

Public Health, Socinstrasse 57, 4002 Basel, Switzerland

Full list of author information is available at the end of the article
} 


\section{Background}

Shrinking the malaria map is now proposed as a realistic goal in many countries [1,2]. The Solomon Islands (SI) are one of the so defined "elimination countries". Being an insular nation with varying levels of endemicity and transmission on most populated islands [3], and located at the margins of the endemic zones makes it feasible to embark upon malaria elimination. However, to reach this target, it is of crucial importance to gather information about the local malaria epidemiology. Limited information is available on the prevalence of mutations associated with drug resistance or on the population structure of malaria parasites in the SI. The present study provides baseline data on Plasmodium falciparum malaria gathered in the Guadalcanal Province in 2004, with focus on anti-malarial resistance.

In vivo studies are still considered the gold standard for the assessment of anti-malarial drug efficacy. The analysis of parasite molecular markers has been proposed as an alternative approach for the estimation of resistance to treatment. It has been shown that chloroquine (CQ) resistance is associated with mutations in pfmdr1 (P. falciparum multidrug resistance gene, coding for P-glycoprotein homologue-1, pgh1) and pfcrt (P. falciparum chloroquine resistance transporter gene) [4-9]. Similarly, it is known that sulphadoxine-pyrimethamine (SP) resistance is associated with mutations in the genes pfdhfr (dihydrofolate reductase) and pfdhps (dihydropteroate synthase) [10-14]. Mutations in pfATPase6 have been described as potential molecular markers involved in resistance to artemisinin derivatives [15]. However, solid evidence for their association with artemisinin resistance is lacking to date.

The analysis of such parasite mutations has the advantage over classical in vivo studies that it can be conducted on samples collected on the first day of health centre attendance. It is therefore independent of compliance and circumvents the need for time- and resourceconsuming follow-up, which often leads to significant patient loss.

Most of the previous studies on parasite mutations were based on samples obtained from patients prior to treatment. However, there is evidence that the molecular profile of parasites circulating in the community matches the one observed among patients attending health centres. Talisuna et al [16] showed that the presence of $p f d h f r$ mutations in the community was independently correlated with the clinical treatment outcome. Therefore, not only symptomatic patients, but also asymptomatic individuals could be used to establish the prevalence of single nucleotide polymorphisms (SNPs) in the parasite population [17].

The classical molecular techniques are based on individual examination of SNPs by restriction fragment length polymorphism (RFLP) analysis, allele-specific amplification or DNA sequencing. Unfortunately, these methods are labour-intensive if performed on many SNPs, especially for the analysis of large sample sizes. The analysis of multiple SNPs has been shown to allow a better prediction of the clinical outcome compared to the evaluation of individual SNPs. This argued for a technique facilitating parallel typing of multiple SNPs [9,18-24]. To enable high throughput analysis of several point mutations, a sensitive and rapid SNP typing technique based on DNA microarray was used for the simultaneous assessment of all known resistanceassociated SNPs located in genes pfcrt, pfmdr1, pfdhfr, pfdhps and pfATPase6 [25].

Prior to this study, the malaria drug resistance microarray was validated with field studies conducted in different epidemiological and geographical settings, including Papua New Guinea (PNG) [17,24], and Tanzania. The relationship between SNP prevalence in samples collected in the asymptomatic community and the rate of treatment failure derived from clinical in vivo studies was investigated. In PNG, two investigated sites had different rates of treatment failure, which were reflected by different patterns of resistance markers in the corresponding parasite populations [17]. In the frame of that multisite study, the present work reports on an additional field study conducted in 2004 at the north of Guadalcanal province in the SI.

A classical in vivo study was conducted to assess the level of $\mathrm{CQ}+\mathrm{SP}$ failure among symptomatic $P$. falciparum malaria patients. In parallel, a cross-sectional survey in the surrounding asymptomatic community allowed the assessment of SNP prevalence. After the completion of this study, the SI Ministry of Health modified the national treatment guidelines for P. falciparum infections. Since 2008, standard first-line treatment for P. falciparum malaria has been changed to the combination of artemether plus lumefantrine.

\section{Methods}

\section{Study area}

The study was conducted in Tetere area, Guadalcanal Province of SI between November 2004 and May 2005, roughly corresponding to the rainy season. The SI are located northeast of Australia, at a latitude of $8^{\circ}$ South and a longitude of $159^{\circ}$ East. The drug efficacy in vivo study was conducted at the Lunga clinic, located about $10 \mathrm{~km}$ east from Honiara, the capital city of the country. The community-based cross-sectional survey was carried out in 3 villages nearby, which are part of the Lunga clinic catchment area. According to the prevalence of malaria among children aged between 2 and 9 years, the endemicity of $P$. falciparum and Plasmodium vivax malaria in that area is considered mesoendemic. 


\section{In vivo drug efficacy study}

Procedures followed the World Health Organization protocol for 28-day assessment of treatment efficacy for uncomplicated $P$. falciparum malaria in areas of low to moderate transmission [26]. Clinically manifest and microscopically confirmed malaria patients visiting the Lunga clinic were recruited if they were aged $>6$ months, had an axillary temperature $\geq 37.5^{\circ} \mathrm{C}$ or history of fever during the last 48 hours, a $P$. falciparum density between 1,000 and 100,000 asexual parasites per microlitre blood, no signs of severe or complicated malaria and no signs of any other disease. The patients received the first dose of medicine on the day of enrolment (i.e. day 0 ). Follow-up visits were scheduled on days 1, 2, 3, 7, 14, 21 and 28. Patients were advised to come to the clinic on any other day if symptoms occurred. On every visit, patients were clinically examined and blood samples were taken by finger prick, except on day 1 . The parasite density was assessed by microscopy after Giemsa staining of blood slides. In case of a microscopically confirmed $P$. falciparum infection on day 0 , standard treatment, which at that time was $C Q+S P$, was administered under supervision. CQ was given on days 0,1 and $2(10 \mathrm{mg} / \mathrm{kg}$ chloroquine phosphate per day) and SP was given as a single dose on day $0(25 \mathrm{mg} / \mathrm{kg}$ sulphadoxine $+1.25 \mathrm{mg} / \mathrm{kg}$ pyrimethamine). Patients failing first-line treatment were treated with quinine (10 $\mathrm{mg} / \mathrm{kg}$ quinine sulphate, three times a day for three days) plus a single dose of SP $(25 \mathrm{mg} / \mathrm{kg}$ sulphadoxine + $1.25 \mathrm{mg} / \mathrm{kg}$ pyrimethamine, on the first day of secondline treatment).

\section{Community-based cross-sectional survey}

Three villages within the catchment area of the clinic have been selected for the community-based cross-sectional survey. Clinical symptoms during the last seven days, history of malaria and consumption of anti-malarials were reported on an individual questionnaire. Axillary temperature was taken with a digital thermometer and blood samples were collected by finger prick. After microscopy examination of the slides, $P$. falciparum positive people were informed of the result and treated with the first-line treatment at that time.

\section{Molecular analyses}

To distinguish a true recrudescence from a new infection (in vivo drug efficacy study), and to determine PCR prevalence of $P$. falciparum infections and the multiplicity of infection (MOI) in the community, paired in vivo study and community survey samples were genotyped by PCR-RFLP of P. falciparum msp 2 (merozoite surface protein 2) [27]. In brief, after DNA extraction (QIAamp DNA Blood Kit, Qiagen, Switzerland), the $m s p 2$ gene was amplified by nested PCR and digested with restriction enzymes Hinf I and Dde I. Restriction fragment patterns were analysed after electrophoresis on $10 \%$ polyacrylamide gels.

Mutations associated with anti-malarial resistance were assessed by microarray technology among P. falciparum positive samples as described in detail elsewhere [25]. The method is based on the parallel PCR amplifications of the target sequences followed by primer extension using fluorochrome-labelled ddNTPs. After denaturation, primer extension products were specifically hybridized onto the microarray. All SNPs assessed on the microarray are listed in Figure 1.

The data output given by the Axon GenePix ${ }^{\mathrm{Tn}}$ software (Molecular Devices Inc., USA) was edited and converted into a table listing all SNPs with their respective genotype (i.e., wild-type, mutated or mixed) for each patient. For quality control of the SNP microarray typing, data of some of the samples were confirmed by sequencing (Macrogen Inc., Korea).

\section{In vivo drug efficacy}

According to the microscopic observation of patient blood smears and after PCR correction by $m s p 2$ genotyping, the individual treatment outcomes were classified according to the categories defined by $\mathrm{WHO}$ for low to moderate transmission areas [26]: Early Treatment Failure (ETF), Late Clinical Failure (LCF), Late Parasitological Failure (LPF), and Adequate Clinical and Parasitological Response (ACPR). The treatment failure rate among $P$. falciparum malaria patients was calculated for patients who successfully completed the study. The overall treatment failure (TF) rate was obtained by pooling ETF, LCF and LPF rates.

\section{Ethical approval}

The National Ethics Committee of the SI and the Basel Ethic Commission (Ethikkommission beider Basel) in Switzerland reviewed and approved the study protocol. All communities and patients (or parents and legal guardians) involved were enrolled in the study after having given informed consent.

\section{Results}

The field procedures were conducted in 2004-2005 in the North coast of Guadalcanal Province in the SI.

\section{In vivo study}

A total of $43(100 \%)$ patients aged between 2 and 23 years (median: 9 years) completed the in vivo study. No patient was lost to follow-up. 18 (41.9\%) patients were included without meeting the required criteria (i.e. they were without fever (or history of fever) and/or had a parasite density below 1,000 P. falciparum/ $\mu$ l on day 0 ). These individuals were censored from further analysis. 


\begin{tabular}{|c|c|c|c|c|c|}
\hline \multicolumn{2}{|r|}{ pfmdr1 } & \multicolumn{2}{|r|}{ pfert } & \multicolumn{2}{|r|}{ pfdhfr } \\
\hline 86 & Asn $->$ Tyr & 72 & Cys $->$ Ser & 16 & Ala $->$ Val \\
\hline 184 & Tyr -> Phe & 74 & Met $->$ Ile & 51 & Asn $->$ Ile \\
\hline 1034 & Ser -> Cys & 75 & Asn -> Asp/Glu & 59 & Cys $->$ Arg \\
\hline 1042 & Asn $->$ Asp & 76 & Lys -> Thr & 108 & Ser $->$ Asn \\
\hline \multirow[t]{9}{*}{1246} & Asp -> Tyr & 97 & His $->$ Gln & $108 \mathrm{~b}$ & Ser -> Thr \\
\hline & & 152 & Thr $->$ Ala & 164 & Ile -> Leu \\
\hline & & 163 & Ser $->$ Arg & & \\
\hline & & 220 & Ala $->$ Ser & & \\
\hline & & 271 & Gln $\rightarrow$ Glu & & \\
\hline & & 326 & Asn $->$ Asp & & \\
\hline & & $326 \mathrm{~b}$ & Asn $->$ Ser & & \\
\hline & & 356 & Ile $->$ Leu & & \\
\hline & & 371 & Arg $->$ Ile & & \\
\hline
\end{tabular}

\begin{tabular}{|l|l|}
\hline \multicolumn{2}{|c|}{ pfdhps } \\
\hline 436 & Ser $->$ Ala/Phe \\
\hline 437 & Ala $->$ Gly \\
\hline 540 & Lys $->$ Glu \\
\hline 581 & Ala $->$ Gly \\
\hline 613 & Ala $->$ Thr \\
\hline 613 b & Ala $->$ Ser \\
\hline 640 & Ile $->$ Phe \\
\hline 645 & His $->$ Pro \\
\hline
\end{tabular}

\begin{tabular}{|l|l|}
\hline \multicolumn{2}{|c|}{ pfATPase6 } \\
\hline 574 & Gln ->Pro \\
\hline 623 & Ala -> Glu \\
\hline 684 & Asn -> Lys \\
\hline 769 & Ser $->$ Asn \\
\hline
\end{tabular}

Figure 1 List of the 34 anti-malarial resistance SNPs assayed on the DNA microarray. CQ resistance-related markers included 5 SNPS located in pfmdr 1 and 13 SNPs located in pfcrt. SP resistance-related markers included 6 SNPs located in pfdhfr and 6 SNPs located in pfdhps. Putative artemisinin-resistance related markers included 4 SNPs located in pfATPase6.

Among the 25 (58.1\%) remaining patients the following outcomes were observed after PCR correction: $22(88 \%)$ ACPR, 1 ETF (4\%) and 2 LPF (8\%), giving a total of $12 \%$ TF.

\section{Community-based cross-sectional survey}

Three villages comprising the catchment area of the Lunga clinic were screened for asymptomatic $P$. falciparum infected individuals. Due to very limited census data from those three villages, the combined total population could only be roughly estimated to 1600 inhabitants. A total of 388 asymptomatic individuals $(24.3 \%$ of the estimated total population) aged between 1 and 73 years (median: 17 years) were screened in the community, among them $53.4 \%$ females. Based on microscopy reading of blood smears, 50/388 (12.9\%) individuals were infected with P. falciparum; using msp2 PCR, 97/ 388 individuals were found positive, giving a prevalence rate of $25 \% P$. falciparum malaria.

RFLP analysis was successfully conducted on 80 samples. The $m s p 2$ PCR fragment revealed $56(70 \%)$ single infections, 18 (22.5\%) double infections, five (6.25\%) triple infections and one (1.25\%) quadruple infection. The mean multiplicity of infection (MOI; mean number of co-infecting parasite clones) in P. falciparum positive samples was 1.39.

Ten PCR reactions were performed for the five genes associated with drug resistance. Of the 97 P. falciparum positive samples analysed, $21(21.6 \%)$ showed poor amplification for the majority of the PCR reactions ( $>5$ poor PCR results out of 10) and had thus to be excluded. The 76 (78.4\%) remaining samples were used for genotyping by microarray. Sixteen unclear microarray typing outcomes were confirmed by sequencing of the corresponding gene fragments (Macrogen Inc., Korea).
Overall, the analysed population showed to be very homogenous (Figure 2). Two different haplotypes were observed for the two CQ resistance-associated genes. With $70 \%$ of the patients having a single infection and the unambiguous DNA microarray signals, it was considered that co-infecting clones had the same pattern of SNPs. The most dominant haplotype showed mutations at positions pfmdr1 N86Y and pfcrt C72 S, N75D/E, K76T, A220 S, N326 D, and I356L, and was observed in $98.4 \%(61 / 62)$ of the population. The second haplotype was observed in one sample only (1.6\%) and differed from the first one by having one additional mutation at position N1042 D in pfmdr1. Other SNPs related to CQ resistance were all wild-type.

For the genes involved in SP resistance, mutations were fixed at positions C59R and S108N in pfdhfr. Other SNPs in $p f d h f r$ and $p f d h p s$ were all wild-type. No mutation was detected in pfATPase6.

\section{Discussion}

The outcome of $\mathrm{CQ}+\mathrm{SP}$ against falciparum malaria was investigated at a clinic located close to Honiara in the SI. At the same time, the presence of resistance-associated SNPs in P. falciparum was assessed in the surrounding asymptomatic community, screened by a cross-sectional survey.

The resistance-associated SNPs observed in the sample set are in line with previous findings from other malaria areas worldwide. Regarding SP resistance, all samples presented two fixed mutations at positions C59R and S108N in pfdhfr and none in pfdhps. The importance of cumulative mutations conferring SP resistance has been described; in particular the combination of the SNPs pfdhfr S108N, N51I, C59R and pfdhps A437G, K540E $[9,28]$ being correlated with anti-folate treatment failure. The double mutant $p f d h f r$ C59R 


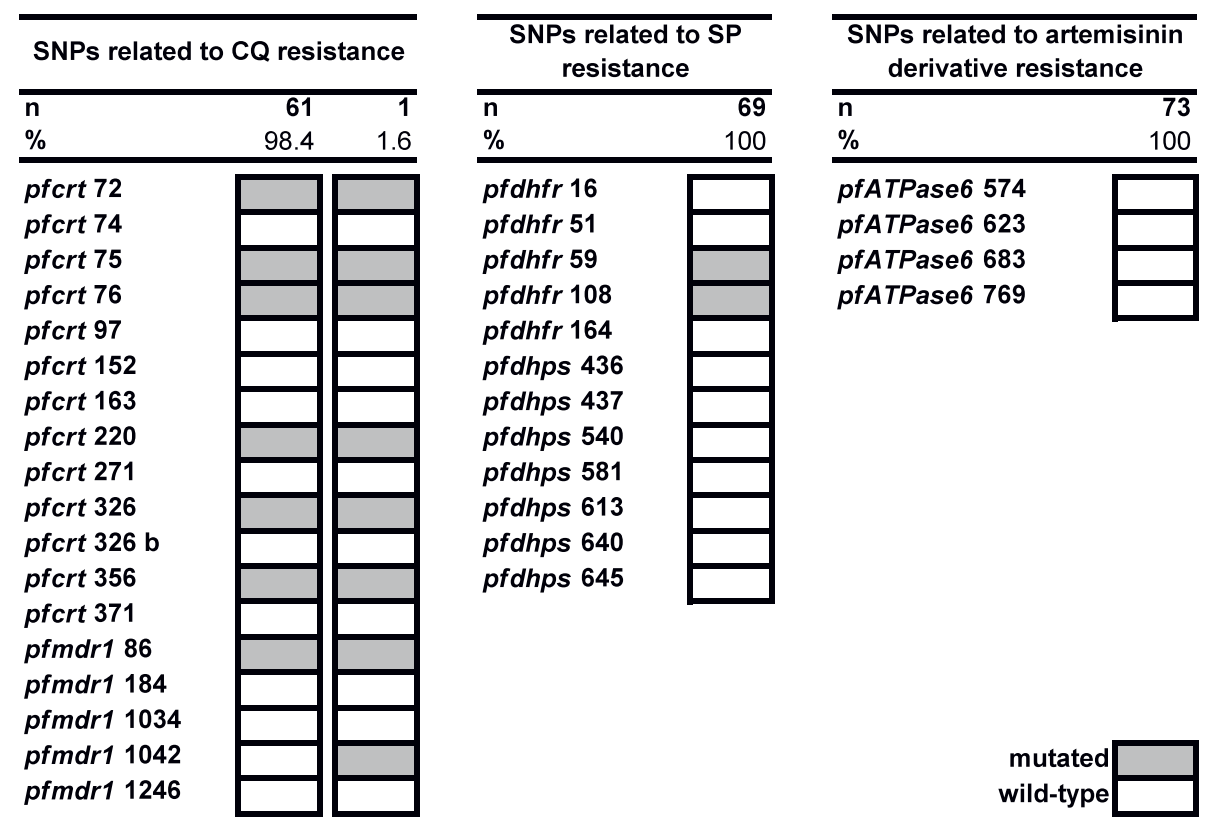

Figure 2 Observed haplotypes of SNPs related to resistance to CQ, SP and artemisinin derivatives. A haplotype is defined by the combination of mutations in genes related to drug resistance. Grey indicates mutations and white indicates wild-type. Two different haplotypes were observed in genes pfmdr1 and pfcrt. One haplotype was observed in genes pfdhfr and pfdhps. One haplotype was observed in gene pfATPase6. $\mathrm{n}$ corresponds to the number of samples assessed for the analysis.

$+\mathrm{S} 108 \mathrm{~N}$, previously shown to be associated with treatment failure [23], has been reported at high prevalence in the SI and other Melanesian countries before $[11,29,30]$. Regarding CQ resistance, fixed mutations at positions pfcrt C72 S, N75D/E, K76T, A220 S, N326 D, I356L and $p f m d r 1 \mathrm{~N} 86 \mathrm{Y}$ were observed. The crucial role of pfcrt $\mathrm{K} 76 \mathrm{~T}$ in conferring CQ resistance is well known, together with additional mutations in $p f c r t$ maintaining functional properties $[5,31]$. The mutation pfmdr1 N86Y is considered as a modulator of CQ resistance in the presence of pfcrt mutations [32-34].

The present study was part of a multisite project simultaneously conducted in Tanzania (Mugittu et al, unpublished) and PNG $[17,24]$. In this context, it is interesting to compare the observations from the SI with reports from PNG due to the close location of both countries (PNG is situated about $1500 \mathrm{~km}$ West of Honiara) and the common origin of the mainly Melanesian human population. The SNPs seen in PNG community samples in 2003-2005 reflected the SI observations with high mutation frequencies in $p f c r t$ and $p f m d r 1$, moderate mutation frequencies in $p f d h f r$, and rare mutations in pfdhps [24]. Also similar to the SI, the mutation $p f m d r 1$ N1042 D was only seen in $1 \%$ of the PNG patients. Mutations in pfATPase6 were neither observed in the SI, nor in PNG. The observation of pfATPase 6 mutations in French Guiana has so far not been confirmed elsewhere $[35,36]$.
Due to the small sample size, conclusions on the correlation between the prevalence of molecular markers in the parasite population infecting the community and the in vivo treatment failure rate cannot be drawn. The in vivo study conducted at the clinic showed $12 \%$ of $C Q$ + SP failure, which can partially be explained by the fixation of the SP resistance-conferring pfdhfr C59R+S108N double mutant in the parasite population. However, PNG data showed that not all mutations predict treatment failure at the same level [24]: mutations pfmdr1 $\mathrm{N} 86 \mathrm{Y}$ and $p f d h p s \mathrm{~A} 437 \mathrm{G}$ were the strongest predictors of amodiaquine+SP failure. It can be postulated that the absence of the pfdhps A437G mutation explains the lower rate of treatment failures in the SI compared to PNG and indicates that SP, and even more specifically sulphadoxine, might have been the effective component of the drug combination. The poor efficacy of CQ has indeed already been reported in the 1980's, when CQ monotherapy was used (Bobogare et al., unpublished data). The combination of CQ+SP used in the $1990 \mathrm{~s}$ as second-line treatment, and subsequently as first-line treatment between 2003 and 2007, certainly further maintained a CQ pressure and thus its limited efficacy.

The use of DNA microarray allowing the parallel analysis of antimalaria drug resistance markers was particularly advantageous in the context of $\mathrm{CQ}+\mathrm{SP}$ as first line treatment, since many polymorphisms have been 
described for those two drugs. In contrast, no marker is validated as indicators of artemether plus lumefantrine combination therapy, which has replaced $\mathrm{CQ}+\mathrm{SP}$ in the SI since 2008. The determination of the $p f m d r 1$ copy number in samples collected after 2008 could provide a useful complement for the assessment of potential resistance to artemether and lumefantrine.

The observed low genetic diversity of the P. falciparum population does probably reflect the context of an island country, where gene flow may be restricted. This low diversity was compared with observations in PNG [24]. In contrast to the SI situation, where mutations associated with antimalarial drug resistance tended to be fixed, the diversity was higher in PNG with various SNP patterns present in the P. falciparum population. However, the most dominant SNP pattern related to CQ resistance seen in PNG between 1992 and 2002 [37] was composed of mutations pfcrt K76T, A220 S, N326 $\mathrm{D}$ and I356L, which corresponds to the dominant haplotype observed in the SI. Regarding SP resistance, the dominant SNP pattern in PNG was the pfdhfr C59R $+\mathrm{S} 108 \mathrm{~N}$ double mutant, which also corresponds to the dominant haplotype in SI.

Parasite populations in SI and PNG show similarities that can be explained by their close geographical vicinity. However, the pronounced insularity and remoteness of the SI differs from PNG and this may be reflected in the lower genetic diversity observed in the SI. The absence of mutations in pfdhps in the SI, which are already present in PNG, is a possible illustration of such low gene flow. A meta-population analysis on the diversity of genes encoding $P$. falciparum vaccine antigens also reported that biogeographic characteristics of island nations in the Pacific constitute a barrier to gene flow [38]. Restricted gene flow is also reflected in the low mean multiplicity of infection in the SI (1.39 concurrent infections in parasite positive samples). For comparison, in a coastal location in PNG having similar geographical characteristics, the multiplicity of infection was slightly higher (1.54), and the $m s p 2$ PCR-based $P$. falciparum prevalence was also higher than in the SI (32\% versus $25 \%$, respectively) [39].

An additional explanation for limited diversity in the considered study site could be the vector control measures. Field evaluations have shown that insecticideimpregnated bed nets and indoor residual spraying together with educational activities significantly reduced malaria transmission in north Guadalcanal Province and contributed to a slow decrease of malaria incidence in the SI since 1992 [40].

To further validate the hypothesis of restricted gene flow in the SI in contrast to PNG, the diversity of various microsatellites which are not under selection pressure could be tested.
In conclusion, the present data provide baseline information on the prevalence of $P$. falciparum drug resistance-associated SNPs and highlight the low level of genetic diversity in the P. falciparum population in the Guadalcanal Province of the SI. Surveillance of molecular markers of drug resistance should be an integral part of the planned malaria eradication programmes, which are currently initiated in insular settings, so that the resistance dynamics can be assessed and the most effective treatment selected.

\section{Acknowledgements}

The fieldwork has been done in collaboration with the SI Malaria Training and Research Institute, based in Honiara. We thank all the study participants in Lunga clinic and surroundings.

For the development of software used for microarray outcome analyses, we want to thank Prof. Thomas Smith and Dr. Nicolas Maire from the Swiss Tropical and Public Health Institute.

The study was supported by grant number 3100-067260 from the Swiss National Science Foundation and by grant number QLK2-CT-2002-01503 from the European Union. Logisitcal support was provided by SIMTRI (Solomon Islands Medical Training and Research Institute).

\section{Author details}

${ }^{1}$ Swiss Tropical and Public Health Institute, Department of Medical Parasitology and Biology of Infection, Department of Epidemiology and Public Health, Socinstrasse 57, 4002 Basel, Switzerland. 'University of Basel, Petersplatz 1, 4003 Basel, Switzerland. ${ }^{3}$ Country Liaison Office, World Health Organization, Honiara, Solomon Islands. ${ }^{4}$ National Vector Borne Diseases Control Programme, Ministry of Health and Medical Services, Honiara, Solomon Islands. ${ }^{5}$ Department of Ambulatory Care and Community Medicine, Infectious Disease Service, University Hospital, Lausanne, Switzerland

\section{Authors' contributions}

MB participated in the field work, conducted the laboratory-based analyses and wrote the manuscript; $J \mathrm{H}$ participated in the study design and field work coordination and edited the manuscript; JM contributed to the molecular analyses and edited the manuscript; AC supervised the microarray analyses; AF participated in the field work; IF helped to draft the manuscript; HPB participated in the study design, supervised the laboratory work and helped to draft the manuscript; BG conceived the study, participated in its design and coordination, and helped to draft the manuscript. All authors read and approved the final manuscript.

\section{Competing interests}

The authors declare that they have no competing interests.

Received: 10 June 2010 Accepted: 6 October 2010

Published: 6 October 2010

\section{References}

1. Richard GA, Feachem AAP, Geoffrey A, on Behalf of The Malaria Elimination Group: Shrinking the Malaria Map - A Prospectus on Malaria Elimination The Global Health Group UCSF Global Health Sciences 2009.

2. Richard GA, Feachem and the Malaria Elimination Group: Shrinking the Malaria Map - A Guide on Malaria Elimination for Policy Makers The Global Health Group UCSF Global Health Sciences 2009.

3. Appleyard B, Tuni M, Cheng Q, Chen N, Bryan J, McCarthy JS: Malaria in pregnancy in the Solomon islands: barriers to prevention and control. Am J Trop Med Hyg 2008, 78:449-454.

4. Foote SJ, Kyle DE, Martin RK, Oduola AM, Forsyth K, Kemp DJ, Cowman AF: Several alleles of the multidrug-resistance gene are closely linked to chloroquine resistance in Plasmodium falciparum. Nature 1990, 345:255-258.

5. Fidock DA, Nomura T, Talley AK, Cooper RA, Dzekunov SM, Ferdig MT, Ursos LM, Sidhu AB, Naude B, Deitsch KW, et al: Mutations in the $P$. 
falciparum digestive vacuole transmembrane protein PfCRT and evidence for their role in chloroquine resistance. Mol Cell 2000, 6:861-871.

6. Sidhu AB, Verdier-Pinard D, Fidock DA: Chloroquine resistance in Plasmodium falciparum malaria parasites conferred by pfcrt mutations. Science 2002, 298:210-213.

7. Duraisingh MT, Cowman AF: Contribution of the pfmdr1 gene to antimalarial drug-resistance. Acta Trop 2005, 94:181-190.

8. Talisuna AO, Bloland P, D'Alessandro U: History, dynamics, and public health importance of malaria parasite resistance. Clin Microbiol Rev 2004 17:235-254.

9. Picot S, Olliaro P, de Monbrison F, Bienvenu AL, Price RN, Ringwald P: A systematic review and meta-analysis of evidence for correlation between molecular markers of parasite resistance and treatment outcome in falciparum malaria. Malar J 2009, 8:89.

10. Triglia T, Menting JG, Wilson C, Cowman AF: Mutations in dihydropteroate synthase are responsible for sulfone and sulfonamide resistance in Plasmodium falciparum. Proc Natl Acad Sci USA 1997, 94:13944-13949.

11. Reeder JC, Rieckmann KH, Genton B, Lorry K, Wines B, Cowman AF: Point mutations in the dihydrofolate reductase and dihydropteroate synthetase genes and in vitro susceptibility to pyrimethamine and cycloguanil of Plasmodium falciparum isolates from Papua New Guinea. Am J Trop Med Hyg 1996, 55:209-213.

12. Plowe CV, Cortese JF, Dimde A, Nwanyanwu OC, Watkins WM, Winstanley PA, Estrada-Franco JG, Mollinedo RE, Avila JC, Cespedes JL, et al: Mutations in Plasmodium falciparum dihydrofolate reductase and dihydropteroate synthase and epidemiologic patterns of pyrimethamine-sulfadoxine use and resistance. J Infect Dis 1997, 176:1590-1596.

13. Triglia T, Wang P, Sims PF, Hyde JE, Cowman AF: Allelic exchange at the endogenous genomic locus in Plasmodium falciparum proves the role of dihydropteroate synthase in sulfadoxine-resistant malaria. Embo J 1998, 17:3807-3815.

14. Gregson A, Plowe CV: Mechanisms of resistance of malaria parasites to antifolates. Pharmacol Rev 2005, 57:117-145.

15. Jambou R, Legrand E, Niang M, Khim N, Lim P, Volney B, Ekala MT, Bouchier C, Esterre P, Fandeur T, Mercereau-Puijalon O: Resistance of Plasmodium falciparum field isolates to in-vitro artemether and point mutations of the SERCA-type PfATPase6. Lancet 2005, 366:1960-1963.

16. Talisuna AO, Langi P, Mutabingwa TK, Watkins W, Van Marck E, Egwang TG, D'Alessandro U: Population-based validation of dihydrofolate reductase gene mutations for the prediction of sulfadoxine-pyrimethamine resistance in Uganda. Trans R Soc Trop Med Hyg 2003, 97:338-342.

17. Marfurt J, Smith TA, Hastings IM, Muller I, Sie A, Oa O, Baisor M, Reeder JC, Beck HP, Genton B: Plasmodium falciparum resistance to anti-malarial drugs in Papua New Guinea: evaluation of a community-based approach for the molecular monitoring of resistance. Malar J 2010, 9:8.

18. Basco LK, Tahar R, Ringwald P: Molecular basis of in vivo resistance to sulfadoxine-pyrimethamine in African adult patients infected with Plasmodium falciparum malaria parasites. Antimicrob Agents Chemother 1998, 42:1811-1814.

19. Basco LK, Tahar R, Keundjian A, Ringwald P: Sequence variations in the genes encoding dihydropteroate synthase and dihydrofolate reductase and clinical response to sulfadoxine-pyrimethamine in patients with acute uncomplicated falciparum malaria. J Infect Dis 2000, 182:624-628.

20. Nzila AM, Mberu EK, Sulo J, Dayo H, Winstanley PA, Sibley CH, Watkins WM: Towards an understanding of the mechanism of pyrimethaminesulfadoxine resistance in Plasmodium falciparum: genotyping of dihydrofolate reductase and dihydropteroate synthase of Kenyan parasites. Antimicrob Agents Chemother 2000, 44:991-996.

21. Kublin JG, Dzinjalamala FK, Kamwendo DD, Malkin EM, Cortese JF, Martino LM, Mukadam RA, Rogerson SJ, Lescano AG, Molyneux ME, et al: Molecular markers for failure of sulfadoxine-pyrimethamine and chlorproguanil-dapsone treatment of Plasmodium falciparum malaria. $J$ Infect Dis 2002, 185:380-388.

22. Pearce RJ, Drakeley C, Chandramohan D, Mosha F, Roper C: Molecular determination of point mutation haplotypes in the dihydrofolate reductase and dihydropteroate synthase of Plasmodium falciparum in three districts of northern Tanzania. Antimicrob Agents Chemother 2003, 47:1347-1354

23. Talisuna AO, Nalunkuma-Kazibwe A, Langi $P$, Mutabingwa TK, Watkins WW Van Marck E, Egwang TG, D'Alessandro U: Two mutations in dihydrofolate reductase combined with one in the dihydropteroate synthase gene predict sulphadoxine-pyrimethamine parasitological failure in Ugandan children with uncomplicated falciparum malaria. Infect Genet Evol 2004, 4:321-327.

24. Marfurt J, Muller I, Sie A, Oa O, Reeder JC, Smith TA, Beck HP, Genton B: The usefulness of twenty-four molecular markers in predicting treatment outcome with combination therapy of amodiaquine plus sulphadoxine-pyrimethamine against falciparum malaria in Papua New Guinea. Malar J 2008, 7:61.

25. Crameri A, Marfurt J, Mugittu K, Maire N, Regos A, Coppee JY, Sismeiro O, Burki R, Huber E, Laubscher D, et al: Rapid microarray-based method for monitoring of all currently known single-nucleotide polymorphisms associated with parasite resistance to antimalaria drugs. J Clin Microbiol 2007, 45:3685-3691.

26. World Health Organization: Assessment and monitoring of antimalarial drug efficacy for the treatment of uncomplicated falciparum malaria. World Health Organization Geneva 2003.

27. Felger I, Beck HP: Genotyping of Plasmodium falciparum. PCR-RFLP analysis. Methods Mol Med 2002, 72:117-129.

28. Sibley CH, Hyde JE, Sims PF, Plowe CV, Kublin JG, Mberu EK, Cowman AF, Winstanley PA, Watkins WM, Nzila AM: Pyrimethamine-sulfadoxine resistance in Plasmodium falciparum: what next? Trends Parasitol 2001, 17:582-588.

29. Mita T, Tanabe K, Takahashi N, Tsukahara T, Eto H, Dysoley L, Ohmae H, Kita K, Krudsood S, Looareesuwan S, et al: Independent evolution of pyrimethamine resistance in Plasmodium falciparum isolates in Melanesia. Antimicrob Agents Chemother 2007, 51:1071-1077.

30. Casey GJ, Ginny M, Uranoli M, Mueller I, Reeder JC, Genton B, Cowman AF: Molecular analysis of Plasmodium falciparum from drug treatment failure patients in Papua New Guinea. Am J Trop Med Hyg 2004, 70:251-255.

31. Cooper RA, Hartwig CL, Ferdig MT: pfcrt is more than the Plasmodium falciparum chloroquine resistance gene: a functional and evolutionary perspective. Acta Trop 2005, 94:170-180.

32. Reed MB, Saliba K, Caruana SR, Kirk K, Cowman AF: Pgh1 modulates sensitivity and resistance to multiple antimalarials in Plasmodium falciparum. Nature 2000, 403:906-909.

33. Babiker HA, Pringle SJ, Abdel-Muhsin A, Mackinnon M, Hunt P, Walliker D: High-level chloroquine resistance in Sudanese isolates of Plasmodium falciparum is associated with mutations in the chloroquine resistance transporter gene pfcrt and the multidrug resistance Gene pfmdr1. J Infect Dis 2001, 183:1535-1538.

34. Chen N, Russell B, Fowler E, Peters J, Cheng Q: Levels of chloroquine resistance in Plasmodium falciparum are determined by loci other than pfcrt and pfmdr1. J Infect Dis 2002, 185:405-407.

35. Tahar R, Ringwald P, Basco LK: Molecular epidemiology of malaria in Cameroon. XXVIII. In vitro activity of dihydroartemisinin against clinical isolates of Plasmodium falciparum and sequence analysis of the $P$. falciparum ATPase 6 gene. Am J Trop Med Hyg 2009, 81:13-18.

36. Zhang G, Guan Y, Zheng B, Wu S, Tang L: No PfATPase6 S769N mutation found in Plasmodium falciparum isolates from China. Malar J 2008, 7:122

37. Nsanzabana C, Hastings IM, Marfurt J, Muller I, Baea K, Rare L, Schapira A, Felger I, Betschart B, Smith TA, et al: Quantifying the evolution and impact of antimalarial drug resistance: drug use, spread of resistance, and drug failure over a 12-year period in Papua New Guinea. J Infect Dis 2010, 201:435-443.

38. Barry AE, Schultz L, Buckee CO, Reeder JC: Contrasting population structures of the genes encoding ten leading vaccine-candidate antigens of the human malaria parasite, Plasmodium falciparum. PLOS One 2009, 4:e8497.

39. Marfurt J: Drug resistant malaria in Papua New Guinea and molecular monitoring of parasite resistance. PhD thesis University of Basel, Swiss Tropical and Public Health Institute 2006.

40. Hii JL, Kanai L, Foligela A, Kan SK, Burkot TR, Wirtz RA: Impact of permethrin-impregnated mosquito nets compared with DDT housespraying against malaria transmission by Anopheles farauti and An. punctulatus in the Solomon Islands. Med Vet Entomol 1993, 7:333-338.

doi:10.1186/1475-2875-9-270

Cite this article as: Ballif et al:: Monitoring of malaria parasite resistance to chloroquine and sulphadoxine-pyrimethamine in the Solomon Islands by DNA microarray technology. Malaria Journal 2010 9:270. 